\title{
Realizing Enhanced Energy Storage and Hardness Performances in 0.90NaNbO3- 0.10Bi(Zn0.5Sn0.5)03 Lead-Free Ceramics
}

\section{Xiaoyan Dong}

Guilin University of Technology

\section{Xu Li}

Sichuan University

Hongyun Chen

Guilin University of Technology

Qinpeng Dong

Guilin University of Technology

Jiaming Wang

Guilin University of Technology

Xiang Wang

Guilin University of Technology

Yue Pan

Guilin University of Technology

Xiuli Chen ( $\nabla$ cxlnwpu@163.com )

Guilin University of Technology

Huanfu Zhou

Guilin University of Technology

\section{Research Article}

Keywords: Energy storage, NaNbO3-based, Domain evolutions, Hardness

Posted Date: August 4th, 2021

DOI: https://doi.org/10.21203/rs.3.rs-746339/v1

License: (c) (i) This work is licensed under a Creative Commons Attribution 4.0 International License.

Read Full License 


\title{
Realizing enhanced energy storage and hardness performances in
}

\section{$0.90 \mathrm{NaNbO}_{3}-0.10 \mathrm{Bi}\left(\mathrm{Zn}_{0.5} \mathrm{Sn}_{0.5}\right) \mathrm{O}_{3}$ lead-free ceramics}

Xiaoyan Dong ${ }^{\mathrm{a}}, \mathrm{Xu} \mathrm{Li}{ }^{\mathrm{b}}$, Hongyun Chen ${ }^{\mathrm{a}}$, Qinpeng Donga ${ }^{\mathrm{a}}$ Jiaming Wang ${ }^{\mathrm{a}}$, Xiang Wang ${ }^{\mathrm{a}}$, Yue Pan ${ }^{\mathrm{a}}$, Xiuli Chen ${ }^{\mathrm{a}, *}$, Huanfu Zhou ${ }^{\mathrm{a},{ }^{*}}$

a Collaborative Innovation Center for Exploration of Hidden Nonferrous Metal Deposits and Development of New Materials in Guangxi, Key Laboratory of Nonferrous Materials and New Processing Technology, Ministry of Education, School of Materials Science and Engineering, Guilin University of Technology, Guilin

$$
\text { 541004, China }
$$

b College of Materials Science and Engineering, Sichuan University, 610064, Chengdu, China

\begin{abstract}
Relaxor behavior has been demonstrated responsible for excellent energy storage characteristics in dielectric materials owing to the fast polarization response, and an ultrahigh energy storage density can also be induced in $\mathrm{NaNbO}_{3}(\mathrm{NN})$-based ceramics via combining antiferroelectric and relaxor features. Most of the existing reports lead-free dielectric ceramics, nevertheless, are still lacking of the relevant research among domain evolution and relaxor behavior. Herein, a novel lead-free solid solution, $(1-x) \mathrm{NaNbO}_{3}-x \mathrm{Bi}\left(\mathrm{Zn}_{0.5} \mathrm{Sn}_{0.5}\right) \mathrm{O}_{3}[x \mathrm{BZS}, x=0.05,0.10,0.15$, and 0.20$]$ ceramics are designed to further illustrate the above issues. Domain evolutions in $x \mathrm{BZS}$ ceramics confirm the contribution of relaxor behavior to the excellent energy storage characteristics, owning to fast polarization rotation based on the low energy barrier of polar nanoregions (PNRs). Consequently, a high energy storage density of $3.14 \mathrm{~J} / \mathrm{cm}^{3}$ and energy efficiency of $83.30 \%$ are simultaneously available at $0.10 \mathrm{BZS}$ ceramics, together with the stabilities of energy storage properties in large temperature range $\left(20-100{ }^{\circ} \mathrm{C}\right)$ and wide frequency range $(1-200 \mathrm{~Hz})$. Additionally, for practical applications, the 0.10BZS ceramics display a great discharge energy storage density $\left(W_{\text {dis }} \sim 1.05 \mathrm{~J} / \mathrm{cm}^{3}\right)$, fast discharge rate $\left(t_{0.9} \sim 60.60 \mathrm{~ns}\right)$, and high hardness $(H \sim 5.49 \mathrm{GPa})$. These *Corresponding authors. E-mail: cxlnwpu@163.com (X. Chen), zhouhuanfu@163.com (H. Zhou).
\end{abstract}


results indicate that this study may provide a considerable new light on the mechanism of high performance lead-free dielectric energy storage materials.

Keywords: Energy storage; $\mathrm{NaNbO}_{3}$-based; Domain evolutions; Hardness 


\section{Introduction}

Dielectric capacitors have broad scope of applications in pulsed power supply devices via virtue of their high power densities, rapid charge/discharge rates, and long cycle lifetimes $[1,2]$. The low energy storage densities, however, limit their further development toward miniaturization and integration. Normally, the energy storage performances (ESP) of dielectric capacitors can be valued via following Eq. (1)-(3) [3-5]:

$$
\begin{gathered}
U_{r e c}=\int_{P_{r}}^{P_{\max }} E d P \\
U=\int_{0}^{P_{\max }} E d P \\
\eta=\frac{U_{\text {rec }}}{U} \times 100 \%
\end{gathered}
$$

where $P_{\max }, P_{\mathrm{r}}, E, U_{\mathrm{rec}}, U$, and $\eta$ are the maximum polarization, remanent polarization, applied electric field, recoverable energy storage density, overall energy storage density, and energy storage efficiency, respectively. Hence, an ideal dielectric capacitor with high $U_{\text {rec }}$ and $\eta$ values should together own a high dielectric breakdown electric field $\left(E_{\mathrm{b}}\right)$ and large polarization difference $\left(\Delta P=P_{\max }-P_{\mathrm{r}}\right)$.

Generally, dielectric capacitors can be divide into four categories: linear dielectrics (LD), ferroelectrics (FE), relaxor ferroelectrics (RFE), and anti-ferroelectrics (AFE) [6-9]. Among these materials, LD possess high $E_{\mathrm{b}}$, but are difficult to obtain large $U_{\text {rec }}$ due to their low dielectric constants [6]. FE own high dielectric constants and moderate $E_{\mathrm{b}}$, but enhanced dielectric nonlinearity and large $P_{\mathrm{r}}$ restraint to the enhancement of $U_{\text {rec }}$ of the lead-free energy storage ceramics [10]. RFE, with high dielectric constants, low $P_{\mathrm{r}}$ and $\operatorname{sim} P-E$ loop are more expected to be utilized for high energy storage [7]. Their high dielectric constants, however, often lead to low $E_{\mathrm{b}}$ of the samples, thus limiting further increases in $U_{\text {rec}}$. Anti-ferroelectrics possess relatively high $\Delta P$, small $P_{\mathrm{r}}$, and moderate $E_{\mathrm{b}}$, which enables a high $U_{\text {rec. }}$ Although a large $U_{\text {rec }}$ is preferable for pulsed power systems, but its low $\eta$ need to be improved [11, 12]. It has recently been shown that the combination of relaxor features and the AFE phase can optimize the AFE-FE phase transition effectively while a high electrical field is induced [13]. An obvious dielectric relaxor characteristic is exhibited when AFE-FE phase transition, which usually caused slender $P-E$ loops. 
Thus, a high $U_{\text {rec }}$ and $\eta$ can be achieved.

Various approaches have been recommended to realized excellent ESP in lead-free dielectric ceramics. For instance, Yang et al. attained a high $U_{\mathrm{rec}}$ of $2.0 \mathrm{~J} / \mathrm{cm}^{3}$ and excellent mechanical properties in $\mathrm{K}_{0.5} \mathrm{Na}_{0.5} \mathrm{NbO}_{3}-\mathrm{BiFeO}_{3}$ ceramics via grain size engineering [14]. Through constructing antiferroelectric and relaxor characteristics, Li et al. achieved both a high $U_{\text {rec }}$ of $2.5 \mathrm{~J} / \mathrm{cm}^{3}$ and $\eta$ of $95.0 \%$ in $\mathrm{Bi}_{0.5} \mathrm{Na}_{0.5} \mathrm{TiO}_{3}-0.45\left(\mathrm{Sr}_{0.7} \mathrm{Bi}_{0.2}\right) \mathrm{TiO}_{3}$ ceramics [13]. Lin et al. fabricated a sandwich structure nanocomposite with heavily loading of one-dimensional $\mathrm{K}_{0.5} \mathrm{Na}_{0.5} \mathrm{NbO}_{3}$ nanofibers and optimized the $E_{\mathrm{b}}$ and $P_{\max }$ synergistically [15]. In addition, some work for $\mathrm{NaNbO}_{3}-\mathrm{BiMeO}_{3}(\mathrm{Me}$ represent trivalent or mean trivalent ions) ceramics have been done in our previous work to realize a high $U_{\text {rec }}\left(>3.0 \mathrm{~J} / \mathrm{cm}^{3}\right)$ and large $\eta(>80.0 \%)$ [16-19]. However, the ceramics in application may be subjected to harsh environments. An abundance of study has been focus on improving ESP but only little attention applying to the hardness. As we all know, the ceramics have a brittle nature due to their strong ionic bonding [20]. When the load is applied, the cracking is generated, but didn't sliding along the slip planes due to the small number of operating slip systems and low density of mobile dislocations. Hardness and a strong microstructure are therefore required to prevent the ceramics from cracking under high frequency vibrations caused by highly applied electric fields or stresses. The internal mechanisms of excellent energy storage characteristics, such as domain evolution, visualized relaxor behavior and the hardness of NN-based ceramics, should be further analyzed.

Herein, a new lead-free ceramic, $(1-x) \mathrm{NaNbO}_{3}-x \mathrm{Bi}\left(\mathrm{Zn}_{0.5} \mathrm{Sn}_{0.5}\right) \mathrm{O}_{3}$ solid solution is designed to illustrate the above problems. Enhanced $\Delta P$ mainly originate from highly dynamic nanodomains, as evidenced by piezoresponse force microscopy (PFM). The enhanced $E_{\mathrm{b}}$ is mainly attributed to the enhanced insulation properties caused by the reduced grain size. In consequence, a high $U$ of $3.14 \mathrm{~J} / \mathrm{cm}^{3}$ and a large $\eta$ of $83.30 \%$ have been simultaneously achieved in 0.10BZS solid solutions. Meanwhile, decent temperature $\left(20-100{ }^{\circ} \mathrm{C}\right)$ and frequency $(1-200 \mathrm{~Hz})$ stabilities of the energy storage characteristics are also achieved. Furthermore, a 
high $W_{\text {dis }}$ of $1.05 \mathrm{~J} / \mathrm{cm}^{3}$, fast discharge time of $60.6 \mathrm{~ns}$ and high hardness of $5.49 \mathrm{GPa}$ also synchronously achieved in this system. These consequences indicate that the 0.10BZS ceramics are promising lead-free material for pulsed power capacitors.

\section{Experimental procedure}

The experimental process and characterization method of $(1-x) \mathrm{NN}-x \mathrm{BZS}$ ceramics are given in the supporting material.

\section{Results and discussion}

XRD patterns tested at room temperature (RT) are exhibited in Fig. 1(a). As $x$ increases, the phase transition, corresponding to from orthorhombic $(O)(x=0.05)$ with the several faintly visible AFE peaks [the red arrows in Fig. 1(a)] to the pseudo-cubic phase $(x \geq 0.05)$, can be verified by the changes in the (110) and (200) diffraction peaks. A similar phenomenon have been reported in other NN-based systems $[16,21]$. Nevertheless, the local scale distortion that exists in the high symmetry phase (cubic) fails to be observed through XRD for the low detection precision. The selected-area electron diffraction (SAED) and high resolution transmission electron microscopy (HR-TEM) patterns of 0.10BZS ceramics were applied to detected the local phase structure, as presented in Fig. 1(b). The interplanar spacing of $d_{(-100)}=0.55(2) \mathrm{nm}$ can be clearly observed via the HR-TEM, inverse Fast Fourier Transformation (FFT), and SAED images, which is conform to the XRD data of the NN ceramics (tetragonal phase, PDF-\#: 73-1106), indicating that the local tetragonal phase is existed in the 0.10BZS ceramics. Particularly, the secondary phases of $\mathrm{Sn}_{2} \mathrm{Nb}_{2} \mathrm{O}_{7}(\sim 2.30 \mathrm{eV})$ [22] and $\mathrm{Bi}_{2} \mathrm{Sn}_{2} \mathrm{O}_{7}(\sim 2.72 \mathrm{eV})$ [23] with low band gap ( 3.20 eV, pure NN [4]) are observed with the BZS content increases. To further confirm the existence of second-phases, Fig. 1(c) and Fig. S1 show the elemental mapping of the 0.20BZS ceramics. The atomic density of the Nb element in $\mathrm{Sn}_{2} \mathrm{Nb}_{2} \mathrm{O}_{7}$ and $\mathrm{Bi}_{2} \mathrm{Sn}_{2} \mathrm{O}_{7}$ are lower than that of $\mathrm{NN}$ ceramics, which causes the content of $\mathrm{Nb}$ missing and minor. In contrast, the $\mathrm{Bi}, \mathrm{Sn}$, and $\mathrm{O}$ elements are enriched in the marked area, resulting higher atom 
densities. These results further verify the existence of the second phases of $\mathrm{Sn}_{2} \mathrm{Nb}_{2} \mathrm{O}_{7}$ and $\mathrm{Bi}_{2} \mathrm{Sn}_{2} \mathrm{O}_{7}$. Generally, the appearance of intrinsic factors such as the low band gap second phase does not immediately deteriorate the $E_{\mathrm{b}}$ of the ceramic [24], and their $E_{\mathrm{b}}$ values are also influenced by extrinsic factors like the grain size and porosity. Herein, the local microstructures and grain size distributions images are performed to further verify the source of high $E_{\mathrm{b}}$, as exhibited in Fig. 1(d). All as-prepared specimens present the dense microstructures and the mean grain size decreases from 3.62 to $2.72 \mu \mathrm{m}$ with increasing $x$. Decreasing the grain size (high densities of the grain boundaries) may be ascribed to the presence of the second phases further inhibits the migration of grain boundary, thereby promoting the increase of $E_{\mathrm{b}}$. In summary, the dense microstructure, small grain size distribution and low content second phase are conducive to improve $E_{\mathrm{b}}$.

Temperature dependent dielectric constant $\left(\varepsilon_{\mathrm{r}}\right)$ and loss $(\tan \delta)$ of the $x \mathrm{BZS}$ ceramics are carried out at various frequencies [Fig. 2(a) and Fig. S2]. A sharp phase transition peak ascribed to the first-order phase transition between the $R$ (AFE, space group Pmnm) and $P$ phases [21], can be observed in 0.05BZS ceramics. With increasing $x$, the temperature of maximum $\varepsilon\left(T_{\mathrm{m}}\right)$ decreased from 243 to $-95{ }^{\circ} \mathrm{C}$, and $\varepsilon_{\mathrm{r}}$ first increased [from 552.56 to 879.41 ] and then decreased [from 879.41 to 639.77] under RT [Fig. 2(b)]. Decreasing $T_{\mathrm{m}}$ is due to the weakened bonding in the $\left[\mathrm{NbO}_{6}\right]$ octahedral [25]. Increasing $\varepsilon_{\mathrm{r}}$ at low $x$ content may be attributed to the $\mathrm{Bi}^{3+}$ ions were dominant [hybridization between the $\mathrm{Bi}^{3+} 6 \mathrm{p}$ and $\mathrm{O}^{2-} 2 \mathrm{p}$ orbitals can cause large polarization] at A-sites. As $x$ further increases, the low-polarization $\left(\mathrm{Zn}^{2+}, \mathrm{Sn}^{4+}\right)$ ions at B-sites will also increase, thereby suppressing the increasing $\varepsilon_{\mathrm{r}}$. In addition, the broadened and weakened dielectric peaks [Fig. S2] provide strong evidence for the enhanced relaxor characteristics, which can also be verified by softened in intensity and broadened in Raman spectroscopy [Fig. S3]. Modified Curie law is employed to further characterize the relaxor behavior of this system and the diffuseness degree $\gamma$ is described by [26, 27]:

$$
\frac{1}{\varepsilon_{r}}-\frac{1}{\varepsilon_{m}}=\frac{\left(T-T_{m}\right)^{\gamma}}{C}
$$

where $\varepsilon_{\mathrm{m}}$ denotes the maximum $\varepsilon_{\mathrm{r}}$ at $T_{\mathrm{m}}, \mathrm{C}$ is the constant. The range of the $\gamma$ value is within 1 (complete FE) 
and 2 (complete RFE). $\gamma$ increased from 1.56 to 1.86 with the increasing BZS [Fig. 2(c)], which is consistent with the above analysis. In $x \mathrm{BZS}$ ceramics, $\mathrm{Bi}^{3+}, \mathrm{Zn}^{2+}$, and $\mathrm{Sn}^{4+}$ ions with various valence and radii respectively hold the A and B-sites of the $\mathrm{NN}$ ceramic, which breaks the long-range order of local electrical fields and local elastic fields, thus causing an enhanced relaxor phenomenon. In short, the ultra-low $\tan \delta$ values $(\sim 0.30 \%)$, large $\varepsilon_{\mathrm{r}}(\sim 879.41)$, and enhanced relaxor characteristics in 0.10BZS ceramics are conducive to achieve excellent ESP.

Fig. 2(d) and (e) show the unipolar $P-E$ loops and energy-storage behaviors of $x \mathrm{BZS}$ ceramics at their $E_{\mathrm{b}}$. Typical AFE characteristics are presented in $0.05 \mathrm{BZS}$ ceramics [Fig. S3], indicating that the introduction of BZS can improve the stability of the AFE phase. Analogous phenomena are also observed in other like-relaxor antiferroelectric ceramics [28]. In AFE ceramics, the polarization directions of adjacent dipoles are opposite, and the dipoles can be reoriented only while a sufficiently large electric field is employed. Most of the AFE domains can turning to long-range ordered FE domains at a large applied electric field, which is extremely conducive to boost $P_{\max }$. Due to the presence of the FE phase, however, their $P_{\mathrm{r}}$ values were large, leading to the low $U_{\text {rec }}$ and $\eta$. With increasing $x$, the enhanced antiferroelectricity and relaxor behavior further alleviate the FE phase in 0.05BZS ceramics [Fig. 2(d)]. As a result, a large $U$ of $3.14 \mathrm{~J} / \mathrm{cm}^{3}$ and high $\eta$ of $83.3 \%$ are acquired in $0.10 \mathrm{BZS}$ ceramics. In order to further measure the energy storage performance of the 0.10BZS ceramics, the stabilities (temperature and frequency) of ceramics are also carried out at $160 \mathrm{kV} / \mathrm{cm}$ and given in Fig. 2(f)-(i). Concerning the temperature stability, as the temperature increased, the $U_{\text {rec }}$ values are gradually decreased from 0.84 to $0.75 \mathrm{~J} / \mathrm{cm}^{3}$ (variation of $U_{\text {rec }} \leq$ $10.72 \%$ ), and the $\eta$ decrease from 86 to $80 \%$. Decreasing $U_{\text {rec }}$ and $\eta$ may be due to the decreasing $P_{\max }$ and heat accumulation at the high temperature. Similarly, 0.10BZS ceramics showed the brilliant frequency stability (variation of $U_{\text {rec }} \leq 4.6 \%$ in $1-200 \mathrm{~Hz}$ ). This satisfactory energy storage stability probably ascribed to the dense microstructure and furthering relaxor behavior in 0.10BZS ceramics [29]. 
For actual applications, charge/discharge behaviors of 0.10BZS ceramics are also assessed. Fig. 3(a) and (b) demonstrate the overdamped discharge curves and $W_{\text {dis }}\left(W_{d i s}=R \int i^{2}(t) d t / V\right.$, where $V$ represents the sample volume and $R(210 \Omega)$ is load resistance) dependence of discharge time under different electrical field of 0.10BZS ceramics. The electrical field dependence of $W_{\text {dis }}$ and $t_{0.9}$ values are introduced in the inset of Fig. 3(a). With increasing the electric field increasing, the $W_{\text {dis }}$ increases from 0.012 to $1.05 \mathrm{~J} / \mathrm{cm}^{3}$, while the small discharge time $t_{0.9}$ has slight fluctuations around 60.6ns. The overdamped discharge curves under different temperature at $180 \mathrm{kV} / \mathrm{cm}$ are shwon in Fig. 3(c). With increasing temperature, the peak current remains a relatively constant value, suggesting the excellent temperature stability of the ceramics [as displayed in inset of Fig. 3(c)]. As it can be observed in Fig. 3(d), as the temperature increases from 20 to $140{ }^{\circ} \mathrm{C}$, the $W_{\text {dis }}$ decreases from 0.78 to $0.69 \mathrm{~J} / \mathrm{cm}^{3}$ (variation less than $12 \%$ ) owing to the changes in $P_{\max }$, and $t_{0.9}$ decreases from 60.2 to $49.8 \mathrm{~ns}$. This excellent charge/discharge behaviors may be mainly due to the enhanced $E_{\mathrm{b}}$ and highly dynamic PNRs [10], indicating that the 0.10BZS ceramics is expected to be a potentially promising candidate for pulse power capacitors.

Besides, the evaluation of hardness properties is also important due to the severe external conditions in practical applications. Fig. 4(a $\left.\mathbf{a}_{1}\right)-\left(\mathbf{a}_{2}\right)$ show the typical load-displacement curves of $x \mathrm{BZS}(x=0.00,0.10)$ ceramics sintered at their optimal sintering temperatures, which is obtained by the nanoindentation test. Obviously, the loading and unloading curves were continuous and smooth for 0.00BZS and 0.10BZS ceramics, indicating that the research components underwent the elastic-plastic deformation without fracturing as the load is applied [30]. The parameters obtained by load-displacement curves, the indentation peak load $(P)$, contact depth $\left(h_{\mathrm{c}}\right)$ contact stiffness ( $S$ is the initial slope of the unloading curve), maximal depth $\left(h_{\mathrm{m}}\right)$ and remaining deformation depth $\left(h_{\mathrm{p}}\right)$, are listed in Table 1. Apparently, the values of $h_{\mathrm{m}}, h_{\mathrm{c}}, h_{\mathrm{p}}$ for $x \mathrm{BZS}(x=0.00,0.10)$ ceramics generally tend to increase as the peak load increases. However, the hardness, obtained by nanoindentation testing, has a tendency to decrease with increasing peak load. It is 
worth noting that the evident hardness of a material obtained directly from nanoindentation are distinct from the real hardness. Proportional Specimen Resistance (PSR) model can be used as an appropriate method to interpret nanoindentation data and describe the relation between effective indentation load and the indentation dimension [31]:

$$
\begin{gathered}
\frac{P_{m}^{\prime}}{h_{c}}=\alpha_{1}+\alpha_{2} h_{c} \\
H_{01}=\frac{P_{m}^{\prime}-\alpha_{1} h_{c}}{24.5 h_{c}^{2}} \\
H_{02}=\frac{\alpha_{2}}{24.5}
\end{gathered}
$$

where $\alpha_{1}$ and $\alpha_{2}$ are two constants for the given materials [the relationship between $P_{\max } / h_{\mathrm{c}}$ and $h_{\mathrm{c}}$ were depicted in Fig. 4( $\left.\mathbf{(} \mathbf{b}_{1}\right)-\left(\mathbf{b}_{2}\right)$, and the coefficient of determination of $R^{2}$ are 0.995 and 0.942$]$, and the value of $H_{01}$ and $H_{02}$ are the real hardness associated to the $\alpha_{1}$ and $\alpha_{2}$, respectively. With increasing peak roads, the $H_{01}$ fluctuates around $H_{02}$. For size effects, the true hardness of the sample differs from the measured hardness. The 3D morphologies of typical pattern of indentations and cracks in BZS ceramics produced by Vickers are shown in Fig. $\mathbf{4}\left(\mathbf{c}_{1}\right)-\left(\mathbf{c}_{2}\right)$. It can be seen that the sample of $x=0.10$ have shorter cracks than $x=0.00$ at the same load. Nevertheless, crack branching, secondary cracking and crack shortening can be clearly found in inset of Fig. 4(c $\left.\mathbf{c}_{\mathbf{1}}\right)$, which might be due to the deteriorating of microstructure. Average length based on diagonals $(d)$ [Fig. S2], hardness can be calculated through the formula below: $H=1.8544 \frac{P_{m}^{\prime}}{d^{2}}$, and the indentation load is $4.903 \mathrm{~N}$. The hardness of $x=0.00$ and $x=0.10$ are $4.68 \mathrm{GPa}$ and $5.49 \mathrm{GPa}$, respectively, indicating an enhancement of hardness, which is consistent with the results of the nanoindentation test. Therefore, the introducing of BZS can effectively improve the hardness of $\mathrm{NaNbO}_{3}$ ceramics.

The impedance spectroscopy is employed to study the conduction mechanism of $x \mathrm{BZS}$ ceramics, as displayed in Fig. 5(a)-(d). Herein, two sets of equivalent random quantum circuits (RQC) [16] were employed to fit the impedance data [see the inset in Fig. 5(a)]. Fig. 5(a)-(d) show decent correlations. 
Generally, the resistance data of the grain and grain boundary conform to the Arrhenius relationship can be expressed as follows [32]:

$$
R=R_{0} \exp \left[E_{\mathrm{rel}} /\left(k_{\mathrm{B}} T\right)\right]
$$

where the value of $R$ is the resistance, $R_{0}$ is the constant, $k_{\mathrm{B}}$ is the Boltzmann constant, and $E_{\text {rel }}$ is the resistance activation energy. The calculated activation energy $\left(E_{\mathrm{g}}\right)$ of the grain in $x \mathrm{BZS}$ solid solutions are $0.73,1.74,0.86$, and $1.20 \mathrm{eV}$, respectively, and the activation energy $\left(E_{\mathrm{gb}}\right)$ of the grain boundary are 1.10 , 1.94, 1.50, $1.72 \mathrm{eV}$, respectively [see Fig. 5(e) and (f)]. Obviously, the $E_{\mathrm{gb}}$ values are larger than that of the $E_{\mathrm{g}}$ for all components, indicating that the grain resistance is dominant at high temperature [33]. Generally, a higher $E_{\mathrm{gb}}$ in $0.10 \mathrm{BZS}$ ceramics means the decrease of free oxygen vacancy concentration at the grain border, i.e., higher barrier of the oxygen vacancy hopping in the grain boundary, which compensate for the grain boundary defect and is beneficial to acquiring high $E_{\mathrm{b}}[18,34]$. Notably, a relatively high $E_{\mathrm{gb}}$ in $0.20 \mathrm{BZS}$ ceramics does not achieve the ideal $E_{\mathrm{b}}$ [see Fig. 2(d)], which may be due to the influence of the second phase [see Fig. 1(a)].

To further reveal the origin of the excellent ESP of $x \mathrm{BZS}$ ceramics, the domain structures of 0.05BZS and $0.10 \mathrm{BZS}$ ceramics are explored according to vertical piezoresponse force microscopy (VPFM) as representatives, as exhibited in Fig. 6. No normal ferroelectric domains are noticed in the amplitude and phase pictures for $0.05 \mathrm{BZS}$ ceramics. This may be attributed to the transition from AFE to FE phase occurring in a high electric field [see Fig. S2] [35], that is, ferroelectric macrodomains with high barriers are dominant in 0.05BZS ceramics [Fig. 6( $\left.\mathbf{c}_{\mathbf{1}}\right)$ and $\left(\mathbf{d}_{\mathbf{1}}\right)$ ]. On the contrary, the nanoscale domains are detected in 0.10BZS ceramics via the comparison of amplitude and phase images [Fig. $\mathbf{6}\left(\mathbf{b}_{1}\right)$ and $\left(\mathbf{b}_{2}\right)$ ]. The incorporation of non-isovalent the $\mathrm{Bi}^{3+}$ and $\left(\mathrm{Zn}^{2+}, \mathrm{Sn}^{4+}\right)$ ions at $\mathrm{A}$ - and B-sites into the host lattice $\mathrm{NN}$ will result in the local charge imbalance and mismatch strain, causing the formation of PNRs and random fields, and some PNRs combine into percolation clusters and then develop into nano-domains [24]. These 
nanodomains have both small hysteresis loss and low switching electrical field because of high dynamics and their low energy barriers [Fig. $\mathbf{6}\left(\mathbf{c}_{2}\right)$ and $\left(\mathbf{d}_{2}\right)$ ], which contribute to achieving weak dielectric nonlinearity and slender $P-E$ hysteresis loops [36].

To better understand the domain structure in $x \mathrm{BZS}$ ceramics, local poling experiments are employed, as illustrated in Fig. 7. A negative DC voltage of $-30.0 \mathrm{~V}$ is employed to the tip of the sample with an region of $3.0 \times 3.0 \mu \mathrm{m}^{2}$, and then different positive voltages $(+7.5,+15.0,+22.5$, and $+30.0 \mathrm{~V})$ are applied on the four square regions of $1.0 \times 1.0 \mu \mathrm{m}^{2}$, respectively, to describe the out-of-plane phase patterns during local poling, as shown in Fig. 7( $\left.\mathbf{a}_{\mathbf{1}}\right)$ and $\left(\mathbf{b}_{\mathbf{1}}\right)$. For $0.05 \mathrm{BZS}$ ceramics, almost no switching domain is observed under +7.5 $\mathrm{V}$ and $+15.0 \mathrm{~V}$. While the poling voltage increases from $+15.0 \mathrm{~V}$ to $+30.0 \mathrm{~V}$, though the switched part of PNRs gradually increases, the switching is yet not complete [Fig. 7( $\left.\left.\mathbf{a}_{1}\right)\right]$. In contrast, a low poling voltage of $+7.5 \mathrm{~V}$ can switch majority of the nanodomains in 0.10BZS ceramics, indicating low switching energy barriers [Fig. 7( $\left.\mathbf{b}_{1}\right)$ ] [37]. Relaxor behavior of the switched domain is also investigated to evaluate the polarization response, as shown in Fig. 7( $\left(\mathbf{a}_{2}\right)-\left(\mathbf{b}_{3}\right)$. The switching domains in 0.05BZS ceramics did not remarkable change after the relaxor time of $30 \mathrm{~min}$. On the contrary, for $0.05 \mathrm{BZS}$ ceramics, the switched nanodomains almost can come back to their initial state in a extremely short time (around 5 min) [Fig. 7( $\left(\mathbf{b}_{2}\right)$ ], indicating highly dynamic nanodomains in 0.10BZS ceramics. Consequently, the nanodomains with low energy barriers and high dynamics are obtained simultaneously, which are facilitate to realizing little $P_{\mathrm{r}}$ and low hysteresis loss, yielding to enhanced ESP.

\section{Conclusions}

A new lead-free relaxor antiferroelectric, NN-based solid solution with enhancement of ESP, has been successfully designed and fabricated via component regulation. Enhancement of ESP are mainly derived from improved $\Delta P$ and $E_{\mathrm{b}}$. Enhancing $\Delta P$ mainly originate from highly dynamic nanodomains, as demonstrated according to piezoresponse force microscopy (PFM). Improving $E_{\mathrm{b}}$ is primarily attributed to 
the synergistic effect of grain size and second phase. Consequently, a large energy storage density of 3.14 $\mathrm{J} / \mathrm{cm}^{3}$, high energy efficiency of $83.30 \%$, and good hardness of 5.49 GPa simultaneously are achieved in 0.10BZS ceramics. Meanwhile, decent thermal $\left(20-100{ }^{\circ} \mathrm{C}\right)$ and excellent frequency $(1-200 \mathrm{~Hz})$ stabilities are also acquired in this system. All presented outcomes suggest that $0.10 \mathrm{BZS}$ ceramics is potentially promising lead-free material for pulsed power capacitors.

\section{Acknowledgement}

This work was supported by Natural Science Foundation of China (Nos. 12064007,11664008 and 6176 1015), Natural Science Foundation of Guangxi (Nos. 2018GXNSFFA050001, 2017GXNSFDA198027 and 2017GXNSFFA198011), High Level Innovation Team and Outstanding Scholar Program of Guangxi Institut es.

\section{CONFLICT OF INTEREST}

The authors declare no conflict of interest.

\section{References}

[1] Wang G, Lu Z, Li Y, et al. Electroceramics for high-energy density capacitors: Current atatus and future perspectives. Chem. Rev. 2021, 121: 6124-6172.

[2] Chu B, Zhou X, Ren K, et al. A dielectric polymer with high electric energy density and fast discharge speed. Science 2006, 313: 334-336.

[3] Zhao P, Cai Z, Chen L, et al. Ultra-high energy storage performance in lead-free multilayer ceramic capacitors via a multiscale optimization strategy. Energ Environ Sci 2020, 13: 4882-4890.

[4] Qi H, Xie A, Tian A, et al. Superior energy-storage capacitors with simultaneously giant energy density and efficiency using nanodomain engineered $\mathrm{BiFeO}_{3}-\mathrm{BaTiO}_{3}-\mathrm{NaNbO}_{3}$ lead-free bulk ferroelectrics. Adv. Energy. Mater. 2019, 10: 1903338-1903345. 
[5] Han K, Luo N, Mao S, et al. Ultrahigh energy-storage density in A-/B-site co-doped $\mathrm{AgNbO}_{3}$ lead-free antiferroelectric ceramics: insight into the origin of antiferroelectricity. J. Mater. Chem. A 2019, 7: 26293-26301.

[6] Luo B, Wang X, Tian E, et al. Enhanced energy-storage density and high efficiency of lead-free $\mathrm{CaTiO}_{3}-\mathrm{BiScO}_{3}$ linear dielectric ceramics. ACS. Appl. Mater. Inter. 2017, 9: 19963-19972.

[7] Dai Z, Xie J, Liu W, et al. Effective strategy to achieve excellent energy storage properties in lead-free $\mathrm{BaTiO}_{3}$-based bulk ceramics. ACS. Appl. Mater. Inter. 2020, 12: 30289-30296.

[8] Luo N, Han K, Cabral MJ, et al. Constructing phase boundary in $\mathrm{AgNbO}_{3}$ antiferroelectrics: pathway simultaneously achieving high energy density and efficiency. Nat Commun 2020, 11: 4824.

[9] Yuan Q, Li G, Yao F-Z, et al. Simultaneously achieved temperature-insensitive high energy density and efficiency in domain engineered $\mathrm{BaTiO}_{3}-\mathrm{Bi}\left(\mathrm{Mg}_{0.5} \mathrm{Zr}_{0.5}\right) \mathrm{O}_{3}$ lead-free relaxor ferroelectrics. Nano. Energy. 2018, 52: 203-210.

[10] Zhou M, Liang R, Zhou Z, et al. Novel $\mathrm{BaTiO}_{3}$-based lead-free ceramic capacitors featuring high energy storage density, high power density, and excellent stability. J. Mater. Chem. C 2018, 6: 8528-8537.

[11] Luo N, Han K, Zhuo F, et al. Aliovalent A-site engineered $\mathrm{AgNbO}_{3}$ lead-free antiferroelectric ceramics toward superior energy storage density. J. Mater. Chem. A 2019, 7: 14118-14128.

[12] Yang D, Gao J, Shu L, et al. Lead-free antiferroelectric niobates $\mathrm{AgNbO}_{3}$ and $\mathrm{NaNbO}_{3}$ for energy storage applications. J. Mater. Chem. A 2020,

[13] Li J, Li F, Xu Z, et al. Multilayer lead-free ceramic capacitors with ultrahigh energy density and efficiency. Adv. Mater. 2018, 30: e1802155.

[14] Yang Z, Gao F, Du H, et al. Grain size engineered lead-free ceramics with both large energy storage density and ultrahigh mechanical properties. Nano. Energy. 2019, 58: 768-777. 
[15] Lin Y, Sun C, Zhan S, et al. Sandwich structure: Ultrahigh discharge efficiency and high energy density in sandwich structure $\mathrm{K}_{0.5} \mathrm{Na}_{0.5} \mathrm{NbO}_{3}$ nanofibers/poly (vinylidene fluoride) composites. Adv Mater Interfaces 2020, 7: 2070047.

[16] Dong X, Li X, Chen X, et al. High energy storage density and power density achieved simultaneously in $\mathrm{NaNbO}_{3}$-based lead-free ceramics via antiferroelectricity enhancement. J. Materiomics. 2020, 7: 629-639.

[17] Dong X, Li X, Chen X, et al. High energy storage and ultrafast discharge in $\mathrm{NaNbO}_{3}$-based lead-free dielectric capacitors via a relaxor strategy. Ceram. Int. 2020, 47: 3079-3088.

[18] Chen H, Shi J, Chen X, et al. Excellent energy storage properties and stability of $\mathrm{NaNbO}_{3}-\mathrm{Bi}\left(\mathrm{Mg}_{0.5} \mathrm{Ta}_{0.5}\right) \mathrm{O}_{3}$ ceramics by introducing $\left(\mathrm{Bi}_{0.5} \mathrm{Na}_{0.5}\right)_{0.7} \mathrm{Sr}_{0.3} \mathrm{TiO}_{3}$. J. Mater. Chem. A 2021, 9: 4789-4799.

[19] Pang F, Chen X, Sun C, et al. Ultrahigh energy storage characteristics of sodium niobate-based ceramics by introducing a local random field. ACS. Sustain. Chem. Eng. 2020, 8: 14985-14995.

[20] Wang X, Venkataraman LK, Tan C, et al. Fracture behavior in electrically poled alkaline bismuth- and potassium- based lead-free piezoceramics using Vickers indentation. Scripta Mater. 2021, 194: 113647.

[21] Zhou MX, Liang RH, Zhou ZY, et al. Superior energy storage properties and excellent stability of novel $\mathrm{NaNbO}_{3}$-based lead-free ceramics with A-site vacancy obtained via a $\mathrm{Bi}_{2} \mathrm{O}_{3}$ substitution strategy. J. Mater. Chem. A 2018, 6: 17896-17904.

[22] Hosogi Y, Shimodaira Y, Kato H, et al. Role of $\mathrm{Sn}^{2+}$ in the band structure of $\mathrm{SnM}_{2} \mathrm{O}_{6}$ and $\mathrm{Sn}_{2} \mathrm{M}_{2} \mathrm{O}_{7}$ $(\mathrm{M}=\mathrm{Nb}$ and $\mathrm{Ta})$ and their photocatalytic properties. Chem. Mater. 2008, 20: 1299-1307.

[23] Hu C-H, Yin X-H, Wang D-H, et al. First-principles studies of electronic, optical, and mechanical properties of $\gamma-\mathrm{Bi}_{2} \mathrm{Sn}_{2} \mathrm{O}_{7}$. Chinese Physics B 2016, 25: 067801. 
[24] Xing J, Yanli H, Wu B, et al. Energy storage behavior in $\mathrm{ErBiO}_{3}$-doped (K,Na) $\mathrm{NbO}_{3}$ lead-free piezoelectric ceramics. ACS Applied Electronic Materials 2020, 2: 3717-3727.

[25] Qu N, Du H,Hao X A new strategy to realize high comprehensive energy storage properties in lead-free bulk ceramics. J. Mater. Chem. C 2019, 7: 7993-8002.

[26] Uchino K,Nomura S Critical exponents of the dielectric constants in diffused-phase-transition crystals. Ferroelectrics 2011, 44: 55-61.

[27] Ji H, Wang D, Bao W, et al. Ultrahigh energy density in short-range tilted NBT-based lead-free multilayer ceramic capacitors by nanodomain percolation. Energy. Storage. Mater. 2021, 38: 113-120.

[28] Ye J, Wang G, Zhou M, et al. Excellent comprehensive energy storage properties of novel lead-free $\mathrm{NaNbO}_{3}$-based ceramics for dielectric capacitor applications. J. Mater. Chem. C 2019, 7: 5639-5645.

[29] Huan Y, Wei T, Wang X, et al. Achieving ultrahigh energy storage efficiency in local-composition gradient-structured ferroelectric ceramics. Chem. Eng. J. 2021, 425. DOI: 10.1016/j.cej.2021.129506.

[30] Xing J, Huang Y, Xu Q, et al. Realizing high comprehensive energy storage and ultrahigh hardness in lead-free ceramics. ACS. Appl. Mater. Inter. 2021, DOI: 10.1021/acsami.1c05153.

[31] Xie S, Xu J, Chen Y, et al. Indentation Behavior and Mechanical Properties of Tungsten/Chromium co-Doped Bismuth Titanate Ceramics Sintered at Different Temperatures. Materials (Basel) 2018, 11: 503.

[32] Longwen $\mathrm{Wu} X W$, Longtu $\mathrm{Li}$ Lead-free $\mathrm{BaTiO}_{3}-\mathrm{Bi}\left(\mathrm{Zn}_{2 / 3} \mathrm{Nb}_{1 / 3}\right) \mathrm{O}_{3}$ weakly coupled relaxor ferroelectric materials for energy storage. RSC.Adv. 2016, 6: 14273-14282.

[33] Li X, Chen X, Zhou H, et al. Phase evolution, microstructure, thermal stability and conductivity behavior of $\left(\mathrm{Ba}_{1-x} \mathrm{Bi}_{0.67 x} \mathrm{~K}_{0.33 x}\right)\left(\mathrm{Ti}_{1-x} \mathrm{Bi}_{0.33 x} \mathrm{Sn}_{0.67 x}\right) \mathrm{O}_{3}$ solid solutions ceramics. J. Alloy. Compd. 2019, 777: 1066-1073. 
[34] Sui J, Fan H, Hu B, et al. High temperature stable dielectric properties and enhanced energy-storage performance of $(1-\mathrm{x})\left(0.85 \mathrm{Na}_{0.5} \mathrm{Bi}_{0.5} \mathrm{TiO}_{3}-0.15 \mathrm{Ba}_{0.8} \mathrm{Ca}_{0.2} \mathrm{Ti}_{0.8} \mathrm{Zr}_{0.2} \mathrm{O}_{3}\right)-\mathrm{xK}_{0.5} \mathrm{Na}_{0.5} \mathrm{NbO}_{3} \quad$ lead-free ceramics. Ceram. Int. 2018, 44: 18054-18059.

[35] Gao J, Zhang Y, Zhao L, et al. Enhanced antiferroelectric phase stability in La-doped $\mathrm{AgNbO}_{3}$ : perspectives from the microstructure to energy storage properties. J. Mater. Chem. A 2019, 7: 2225-2232.

[36] Li X, Chen X, Sun J, et al. Novel lead-free ceramic capacitors with high energy density and fast discharge performance. Ceram. Int. 2020, 46: 3426-3432.

[37] Yin J, Li C, Li R, et al. Defects-induced superior piezoelectric response in perovskite $\mathrm{KNbO}_{3}$. J. Eur. Ceram. Soc. 2020, 41: 2506-2513. 
Table 1 Parameters of $x$ BZS $(x=0.00$ and 0.10$)$ ceramics derived from nanoindentation test.

\begin{tabular}{|c|c|c|c|c|c|c|c|c|c|c|}
\hline Component & $\begin{array}{c}P_{\mathrm{m}} \\
(\mathbf{G P a})\end{array}$ & $\begin{array}{c}h_{\mathrm{m}} \\
(\mathrm{nm})\end{array}$ & $\begin{array}{c}\boldsymbol{h}_{\mathrm{c}} \\
(\mathbf{n m})\end{array}$ & $\begin{array}{c}h_{\mathrm{p}} \\
(\mathrm{nm})\end{array}$ & $S$ & $\begin{array}{c}H \\
\text { (GPa) }\end{array}$ & $\begin{array}{c}\alpha_{1} \\
(\mathbf{m N} / \mathbf{n m})\end{array}$ & $\begin{array}{c}\alpha_{2} \\
\left(\mathrm{nN} / \mathbf{n m}^{2}\right)\end{array}$ & $\begin{array}{c}\boldsymbol{H}_{01} \\
(\mathbf{G P a})\end{array}$ & $\begin{array}{r}H_{02} \\
(\mathbf{G P a})\end{array}$ \\
\hline \multirow[t]{4}{*}{0.00} & 9.988 & 236 & 180 & 160 & 0.0260 & 8.82 & 0.0254 & 0.000167 & 6.82 & 6.81 \\
\hline & 19.994 & 350 & 278 & 250 & 0.0669 & 8.35 & & & 6.83 & 6.81 \\
\hline & 29.94 & 428 & 345 & 300 & 0.1310 & 8.31 & & & 7.26 & 6.81 \\
\hline & 39.922 & 509 & 410 & 365 & 0.2151 & 7.85 & & & 7.16 & 6.81 \\
\hline \multirow[t]{4}{*}{0.10} & 9.993 & 227 & 173 & 155 & 0.247 & 9.81 & 0.0158 & 0.000243 & 9.99 & 9.92 \\
\hline & 19.984 & 335 & 256 & 230 & 0.0617 & 9.18 & & & 9.93 & 9.92 \\
\hline & 29.999 & 428 & 338 & 295 & 0.1215 & 9.03 & & & 8.81 & 9.92 \\
\hline & 39.978 & 508 & 412 & 365 & 0.2326 & 8.55 & & & 8.05 & 9.92 \\
\hline
\end{tabular}




\section{Figure captions:}

Fig. 1 (a) XRD patterns and the enlargement images of $x$ BZS ceramics. (b) SEM of $x$ BZS ceramics. (c) The SAED and HR-TEM images of 0.10BZS ceramics (Inset shows the local inverse Fast Fourier Transform image). (d) The elemental mapping images of 0.20 BZS ceramics.

Fig. 2 (a) The $\varepsilon_{\mathrm{r}}$ and $\tan \delta$ of $x \mathrm{BZS}$ ceramics as a function of temperature, and $f=10 \mathrm{kHz}$. (b) and (c) The $\varepsilon_{\mathrm{r}}$, $T_{\mathrm{m}}$ and $\gamma$ versus the $x$ for $x \mathrm{BZS}$ ceramics. (d) Unipolar $P-E$ loops at various electrical fields of $x \mathrm{BZS}$ ceramics. (e) The $U_{\text {rec }}, U$ and $\eta$ for $x$ BZS ceramics at critical electric fields. (f)-(i) The stabilities (temperature and frequency) of 0.10BZS ceramics.

Fig. 3 (a) Overdamped discharge waveforms, (b) variation of $W_{\mathrm{D}}$ for $0.10 \mathrm{BZS}$ ceramics versus various electric field. (c) Overdamped discharge waveforms, (d) corresponding $W_{\mathrm{D}}$ and $t_{0.9}$ for $0.10 \mathrm{BZS}$ ceramics at various temperature under applied electric field of $180 \mathrm{kV} / \mathrm{cm}$

Fig. $4\left(a_{1}\right)-\left(a_{1}\right)$ Load-displacement curves at different peak loads; $\left(b_{1}\right)-\left(b_{2}\right) P_{\max } / h_{c}$ as a function of $h_{c}$; $\left(c_{1}\right)-\left(c_{2}\right)$ The 3D morphologies of Vickers indentation and cracks of $x$ BZS $(x=0.00,0.10)$ ceramics.

Fig. 5 (a)-(d) Complex impedance plots of the $x$ BZS ceramics measured under different temperatures from 480 to $560^{\circ} \mathrm{C}$. (e) The Arrhenius fitting of grain and grain boundary resistance data of $x \mathrm{BZS}$ ceramics. (f) Changes in $E_{\mathrm{g}}$ and $E_{\mathrm{gb}}$ at different components.

Fig. 6 (a) Schematic diagram of detailed domain structure variation of the process.

Fig.7 (a) PFM amplitude and phase pictures after poling treatment with varying voltages and relaxor periods of (a) 0.05BZS and (b) 0.10BZS ceramics. 

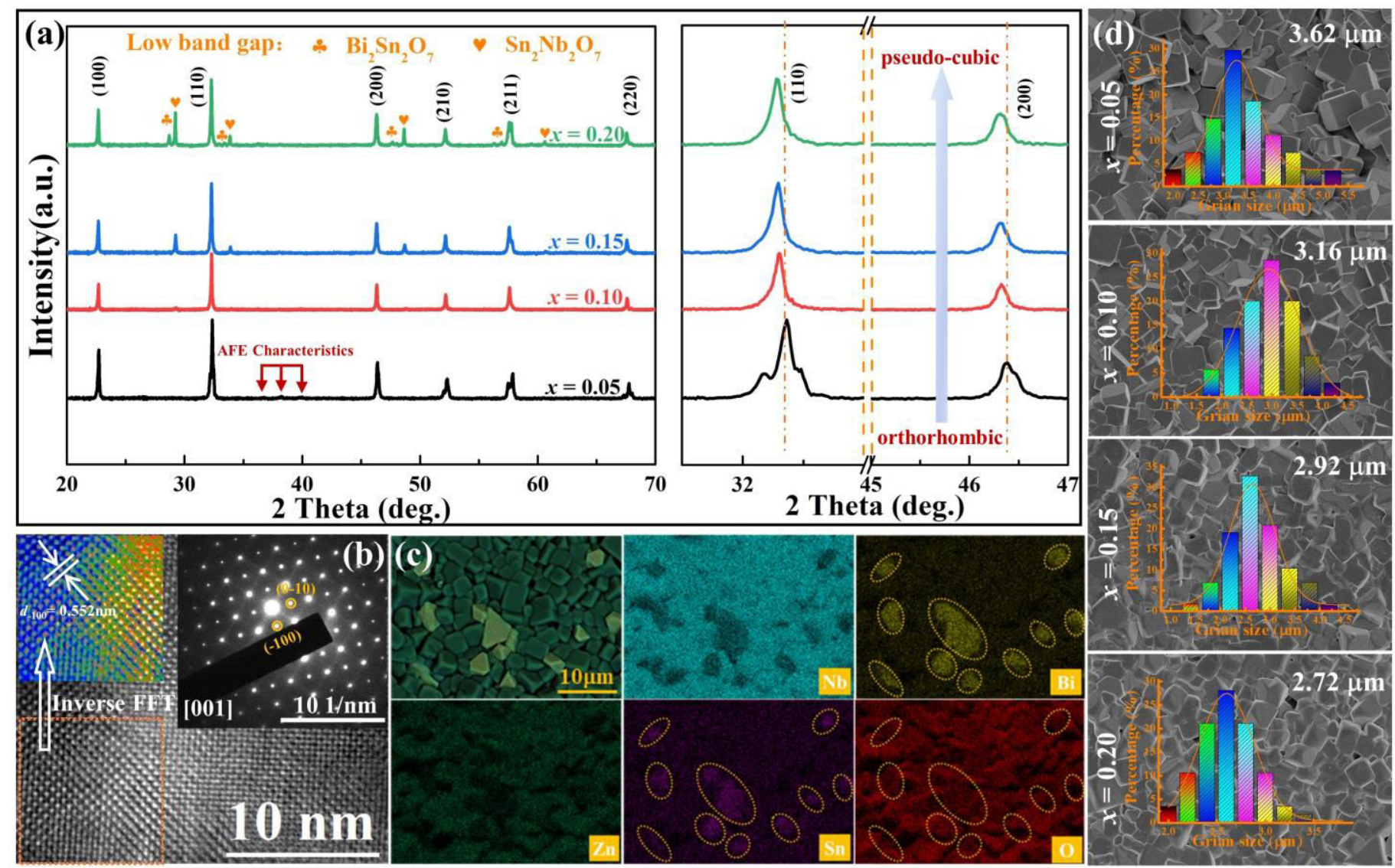

Fig. 1 

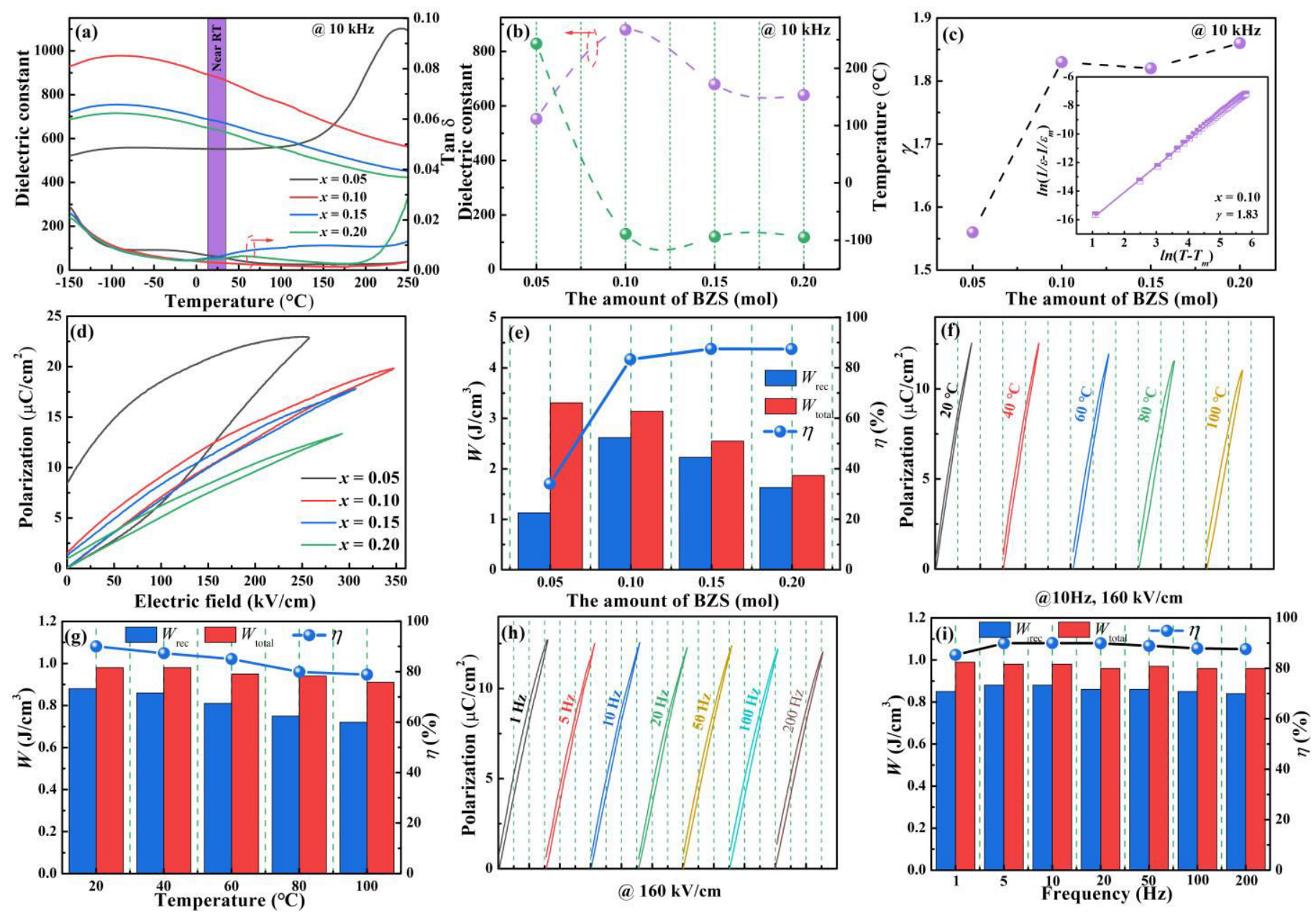

Fig. 2 

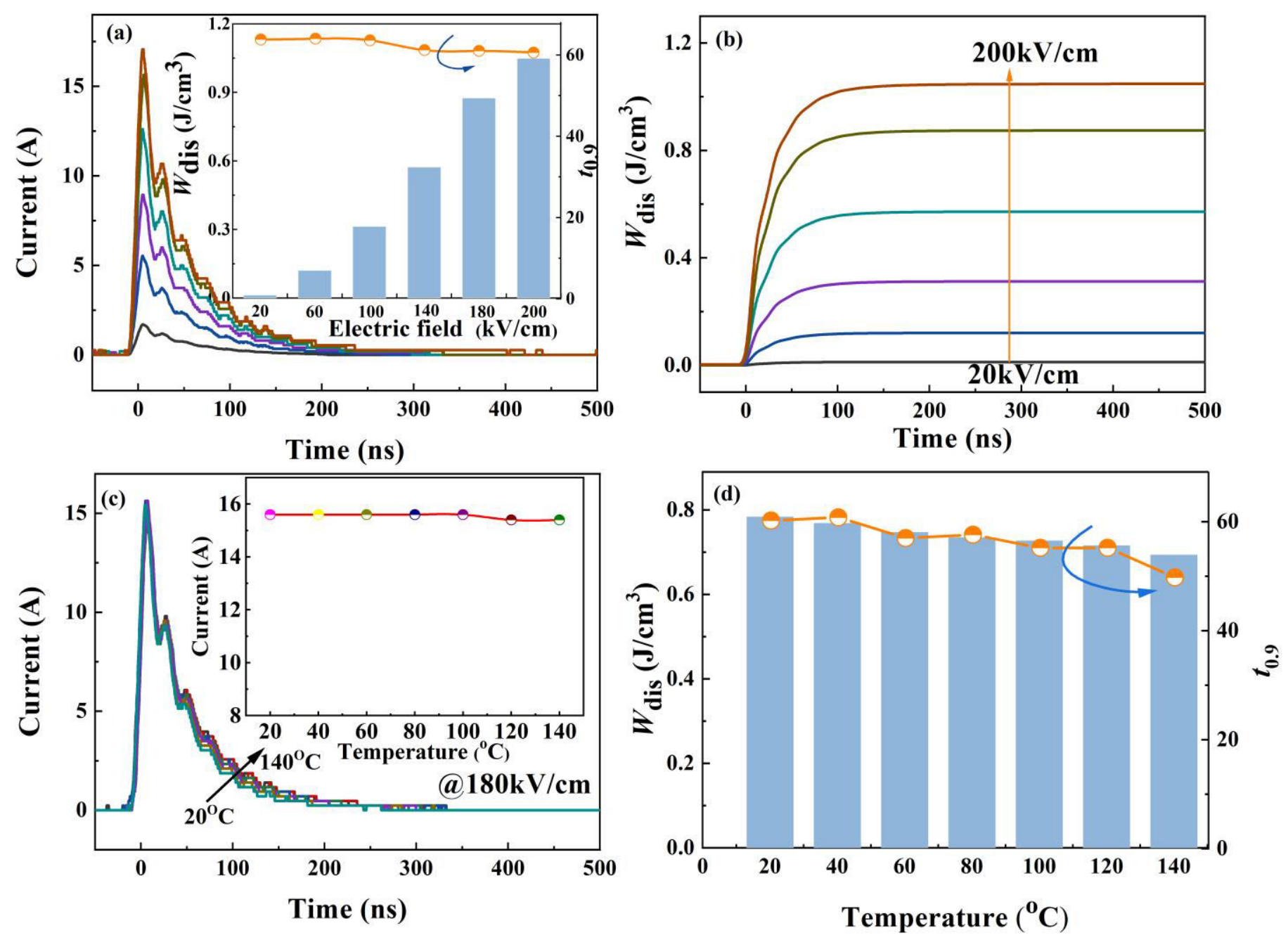

Fig. 3 

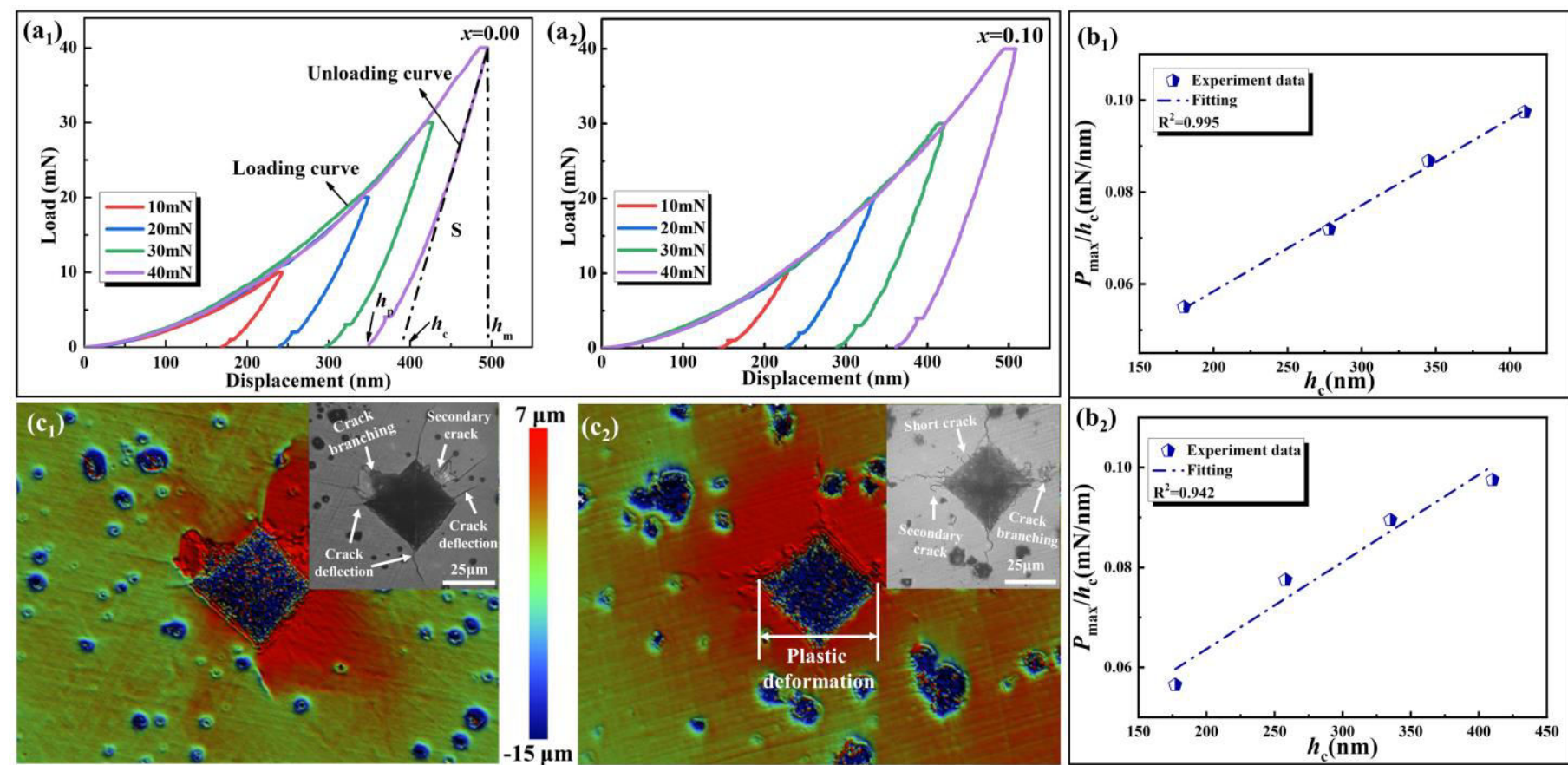

Fig. 4 

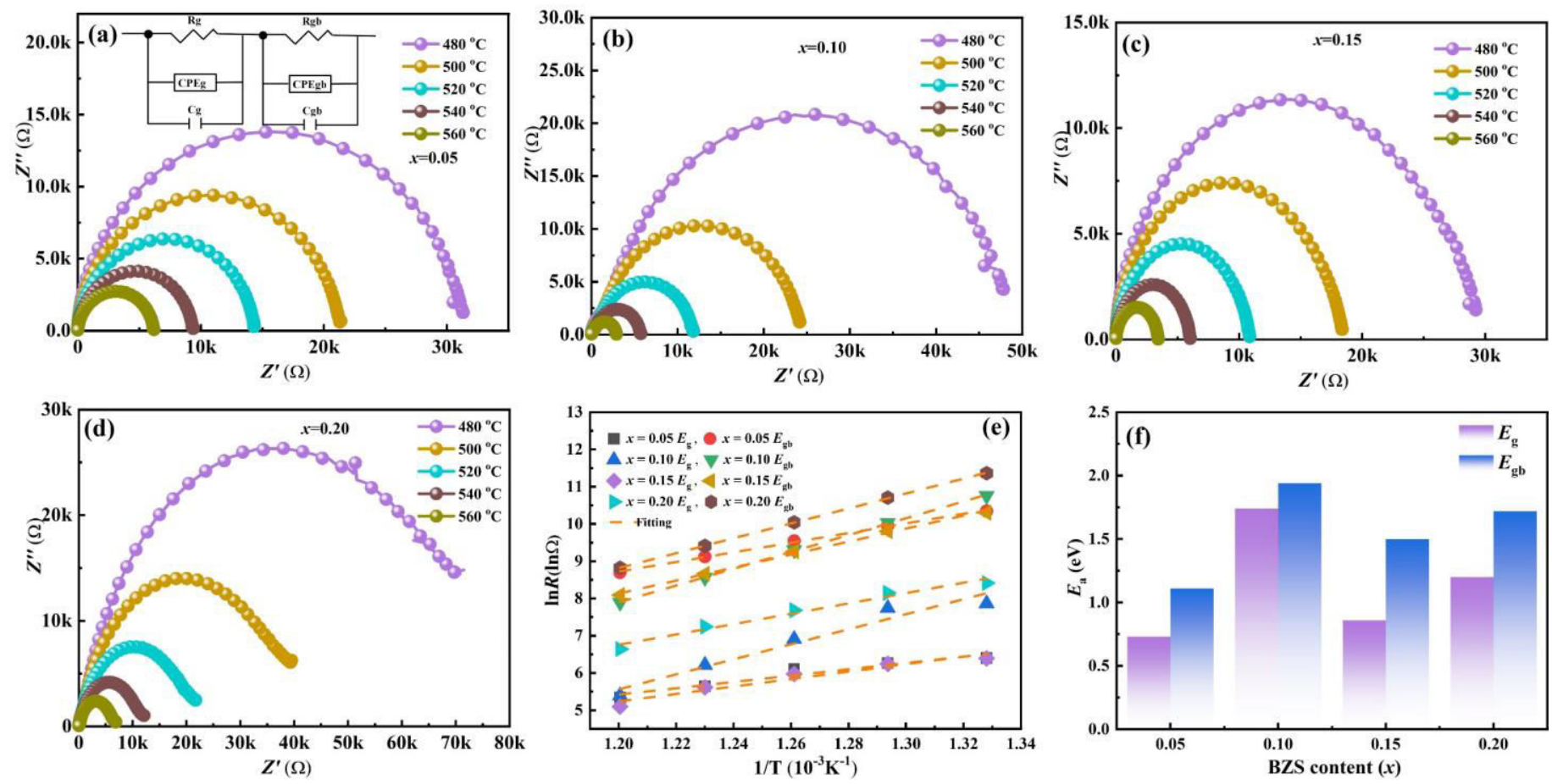

Fig. 5 


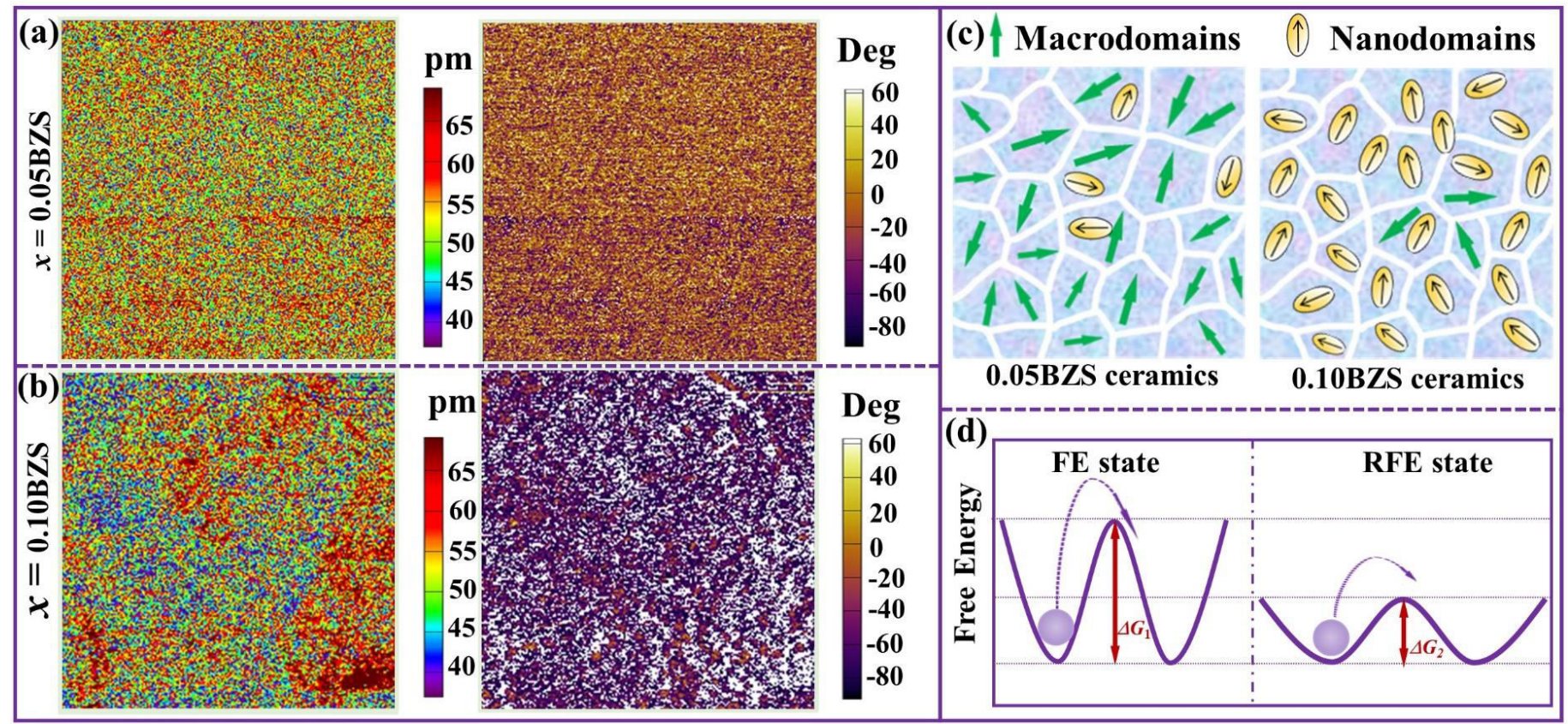

Fig. 6 


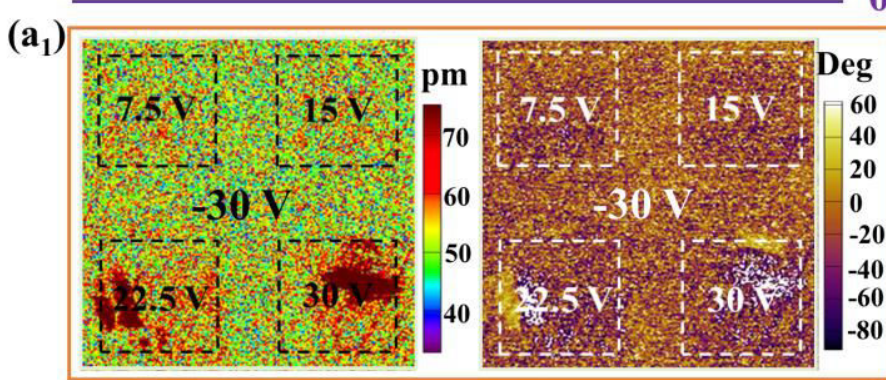

0 min
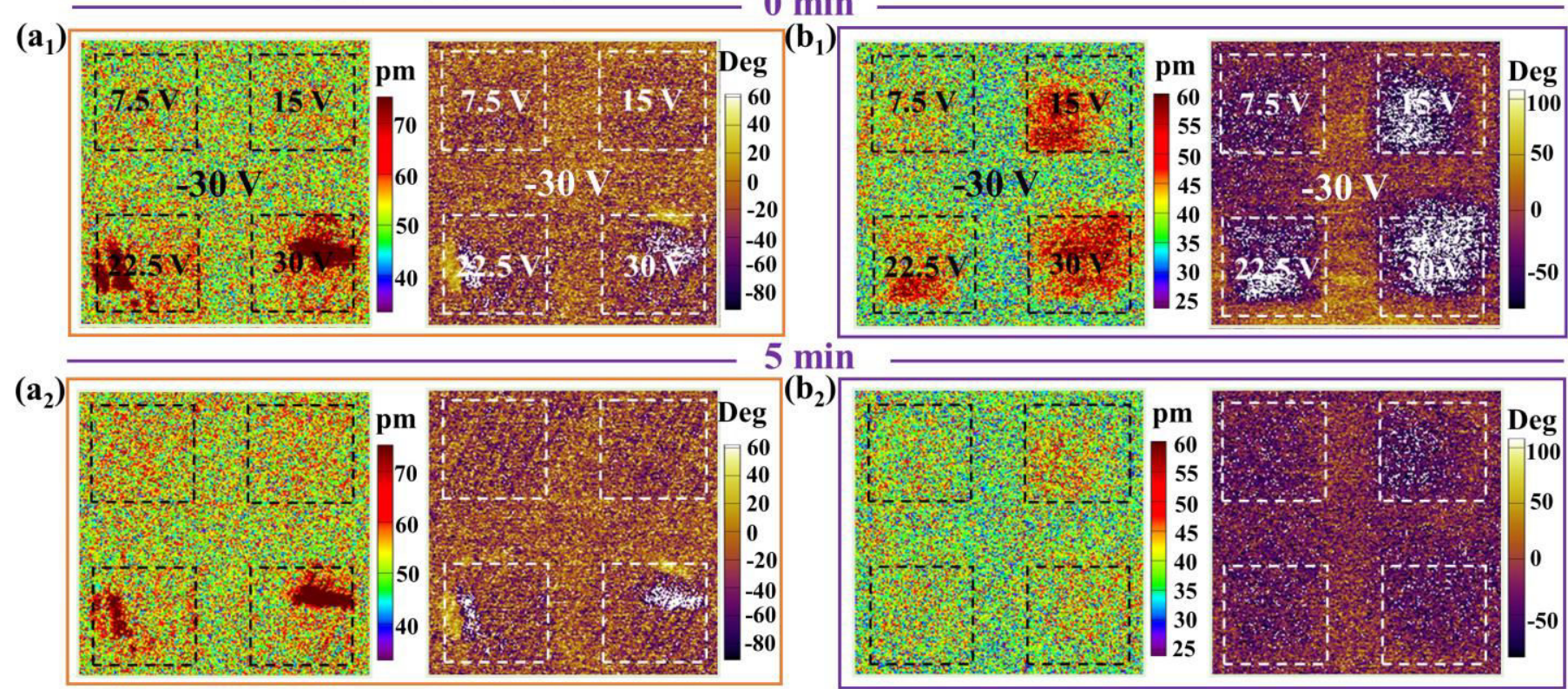

5 min
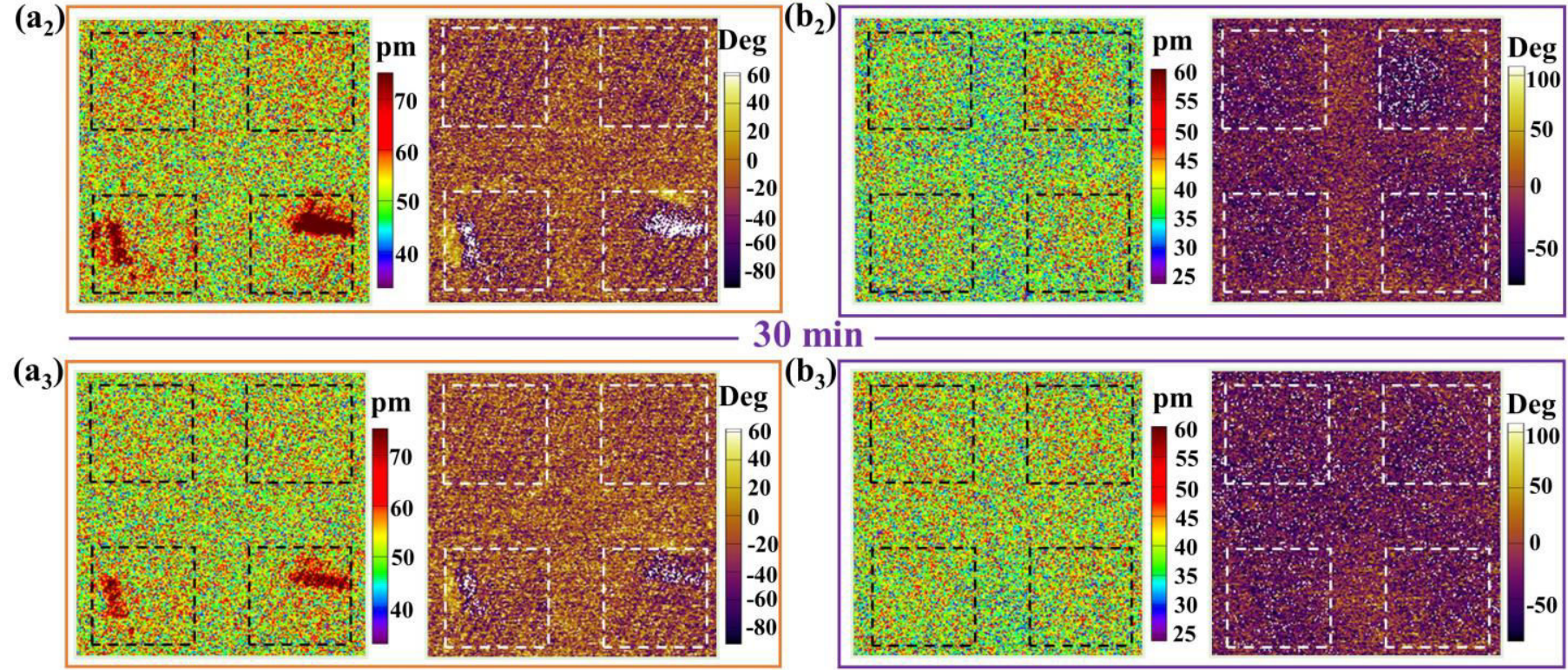

Fig. 7 


\section{Supplementary Files}

This is a list of supplementary files associated with this preprint. Click to download.

- SupplementaryMaterial.doc 